\title{
Downregulation of Sirt1 as aging change in advanced heart failure
}

\author{
Tse-Min Lu, ${ }^{1,2}$, Jia-Yun Tsai ${ }^{3}$, Yen-Chung Chen ${ }^{1,4}$, Chun-Yang Huang ${ }^{1,5}$, Hung-Lung Hsu ${ }^{1,3}$, Chi-Feng Weng ${ }^{1}$,
} Chun-Che Shih ${ }^{1,3}$ and Chiao-Po Hsu ${ }^{1,3^{*}}$

\begin{abstract}
Background: In congestive heart failure the balance between cell death and cell survival in cardiomyocytes is compromised. Sirtuin 1 (Sirt1) activates cell survival machinery and has been shown to be protective against ischemia/reperfusion injury in murine heart. The role of Sirt1 in heart failure, especially in human hearts is not clear.

Results: The expression of Sirt1 and other (associated) downstream molecules in human cardiomyocytes from patients with advanced heart failure was examined. Sirt1 was down-regulated $(54.92 \% \pm 7.80 \%$ in advanced heart failure samples compared with healthy control cardiomyocytes). The modulation of molecules involved in cardiomyocyte survival and death in advanced heart failure were also examined. The expression of Mn-superoxide dismutase and thioredoxin 1, as well as an antiapoptotic molecule, BCl-xL, were all significantly reduced in advanced heart failure cardiomyoctes ( $0.71 \pm 0.02$-fold, $0.61 \pm 0.05$-fold, and $0.53 \pm 0.08$-fold vs. control, respectively); whereas the expression of proapoptotic molecule Bax was significantly increased (1.62 \pm 0.18 -fold vs. control). Increased TUNEL-positive number of cardiomyocytes and oxidative stress, confirmed by 8-hydorxydeoxyguanosine staining, were associated with advanced heart failure. The AMPK-Nampt-Sirt1 axis also showed inhibition in advanced heart failure in addition to severely impaired AMPK activation. Increased p53 (acetyl form) and decreased FoxO1 translocation in the nucleus may be the mechanism of down-regulation of antioxidants and up-regulation of proapoptotic molecules due to low expression of Sirt1.
\end{abstract}

Conclusion: In advanced heart failure, low Sirt1 expression, like aging change may be a significant contributing factor in the downregulation of antioxidants and upregulation of proapoptotic molecules through the p53, FoxO1, and oxidative stress pathways.

Keywords: Aging, Heart failure, Sirt1

\section{Background}

Despite recent progress, congestive heart failure remains a major cause of death in Western countries. The prevalence of congestive heart failure (CHF) increases with age [1]. Due to the increased average life expectancy over the past decade and the increased proportion of aged individuals, it is expected that CHF diagnoses will increase steeply in the near future.

There has been great progress towards understanding CHF pathogenesis and the implications of aging. Increasing

\footnotetext{
* Correspondence: chiaopo@ms39.hinet.net

'National Yang-Ming University, Institute of Clinical Medicine, School of Medicine, Taipei, Taiwan

${ }^{3}$ Division of Cardiovascular Surgery, Department of Surgery, Taipei Veterans General Hospital, 201, Section 2, Shih-Pai Road, Taipei 112, Taiwan Full list of author information is available at the end of the article
}

evidence suggests that the progression of heart failure is determined by the imbalance between cell death promotion and cell survival/protective mechanisms [2]. In CHF or in an aging heart a predominant factor is the role of cell death [3].

Sirtuin 1 is a member of the Sirtuin family of class III histone deacetylases. Sirt1 is involved in gene silencing, longevity, cellular senescence, cell differentiation, and cell survival $[4,5]$. It has been shown that Sirt1 activity enhances the lifespan of several organisms, including yeast, C. elegans, and metazoan [6-8]. The protein expression of Sirt1 in the heart has been demonstrated to be the highest in the embryo with progressive reductions associated with aging [9]. These observations imply that Sirt1 may play an important role in the aging process, and with cardiomyocyte death and survival. 
Sirt1 has been shown to inhibit apoptosis and to delay changes associated with aging in cardiomyocytes using a transgenic mouse as the model [10]. We have also found that Sirt1 expression was significantly reduced in hearts subjected to ischemic/reperfusion. Preconditioning before ischemic/reperfusion increased Sirt1 expression, and was associated with the upregulation of antioxidants and downregulation of proapoptotic molecules, partly through the activation of FoxO1 and reduced oxidative stress [11]. In vitro, endogenous Sirt1 has been found to play an essential role in mediating cardiomyocyte survival: overexpression of Sirt1 has provided protective effects for cardiomyocytes from apoptosis, whereas downregulation of Sirt1 may be associated with apoptotic cell death [12]. However, expression of Sirt1 in advanced heart failure models is not clear and remains controversial. Pillai et al. showed that Sirt1 is down-regulated in heart failure from human samples [13]: however, Alcendor et al. showed that Sirt1 is up-regulated after 4 weeks of pressure overload in mice, a condition used to represent the stage of heart failure [10].

In CHF, the ventricle or atrium wall tension is increased which subjects the cardiomyocytes to higher pressure and relative ischemia compared with normal healthy control hearts. It is implied that the molecular change following ischemia or ischemic/reperfusion in the mouse heart could be applied to the condition of CHF in human beings. Therefore, the goal of this study was to determine the expression of Sirt1 and the associated molecules involved in cell death or survival in a human model.

\section{Methods}

\section{Collection of cardiac samples}

This study was performed according to the guidelines of the Declaration of Helsinki. All procedures involved human tissue were approved by the institutional review boards of the Taipei Veterans General Hospital. Consent was obtained from patients or their families before tissue procurement. Myocardial samples over the posterior wall of the left atrium were collected from donors while being prepared for transplantation. Myocardial samples near the mitral annulus were obtained from patients at the time of therapeutic transplantation. Samples for biochemical analyses were stored at $-80^{\circ} \mathrm{C}$ immediately after procurement. Selected samples for immunostaining were fixed in $4 \%$ paraformaldehyde in phosphate-buffered saline ( $\mathrm{pH} 7.4)$, paraffin-embedded, and sectioned. C57BL/6JNarl mice were obtained from National Laboratory Animal Center of Taiwan, and the investigation conformed with the Guide for the Care and Use of Laboratory Animals published by the US National Institutes of Health.

\section{Cell cultures and materials}

H9c2 cells (rat cardiomyoblasts), obtained from ATCC (Manassas, VA), and were cultured in DMEM supplemented with $10 \%$ fetal bovine serum under an atmosphere of $95 \%$ air $/ 5 \% \mathrm{CO}_{2}$ at $37^{\circ} \mathrm{C}$. Compound $\mathrm{C}$ (Merck) was added to the medium for the times and at the doses indicated. The antibodies used included Sirt1, 8-OHdG goat polyclonal (Millipore), MnSOD (Upstate Biotechnology), Sirt1 rabbit polyclonal, Trx1, Troponin C mouse monoclonal (Santa Cruz), p53, Acetyl-p53 (Lys379), AMPK $\alpha$ and phosphoAMPK $\alpha(T h r 172)$ (Cell Signaling), Nampt (Bethyl), Bcl-xL (Pharmingen), Bax (Abcam), FoxO1 rabbit monoclonal (Epitomics), and tubulin (Sigma).

\section{Immunoblot analysis}

Heart samples were placed in lysis buffer $(50 \mathrm{mmol} / \mathrm{L}$ Tris- $\mathrm{HCl}$ pH 7.4, 0.1\% SDS, $1 \% \mathrm{NP} 40,0.15 \mathrm{~mol} / \mathrm{L} \mathrm{NaCl}$, $0.25 \% \mathrm{Na}$-deoxycholate, and $1 \mathrm{mmol} / \mathrm{L}$ EDTA supplemented with protease inhibitors). Densitometric analysis was performed using Scion Image software (Scion).

\section{Immunohistochemistry and immunofluorescence}

The paraffin-embedded heart specimens, sectioned at 4$\mu \mathrm{m}$ thickness, were deparaffinized and rehydrated in PBS. Pretreatments included microwave antigen retrieval in a $10 \mathrm{mM}$ citrate buffer for $20 \mathrm{~min}$. Immunohistochemical staining was performed using an immunocruz staining system (streptavidin-biotin peroxidase method), and sections were counterstained with hematoxylin. Immunofluorescence analysis was achieved using primary antibodies of Sirt1 (Santa Cruz), FoxO1 (Epitomics), acetyl-p53 (Cell Signaling), and/or Troponin C (Santa Cruz) after a blocking step with $3 \%$ BSA for 30 minutes. DyLight ${ }^{\text {tm }} 488$-conjugated and/or "m594-conjugated affinipure IgG (Thermo Fisher Scientific) were used for secondary antibodies.

\section{Evaluation of apoptosis in tissue sections}

Deoxyribonucleic acid (DNA) fragmentation was detected in situ using TUNEL Briefly, deparaffinized tissue sections were incubated with proteinase $\mathrm{K}$, and DNA fragments were labeled with fluorescein-conjugated dUTP using TdT (Roche Molecular Biochemicals). Myocyte was identified by Troponin $\mathrm{C}$ antibody. Cell nuclei were counterstained with DAPI (blue).

\section{Statistics}

All values were expressed as mean \pm SEM. Statistical analyses between groups were done using a one-way ANOVA, and when $\mathrm{F}$ values were significant at a $95 \%$ confidence limit, differences among group means were evaluated using Fisher's project least significant difference post-test procedure for group data. A P value less than 0.05 was considered significant.

\section{Results}

Totally, the cardiac tissue samples were harvested from 10 donors (eight males, aged $37.5 \pm 3.9$ years) and 10 
recipients of dilated cardiomyopathy (six males, aged $40.1 \pm 3.7$ years, $P=0.637$ ). The cardiothoracic ratio of recipients was $67.65 \pm 1.06 \%$, measured by posteroanterior film of chest $\mathrm{x}$ ray.

\section{Sirt1 is down-regulated in advanced heart failure of human beings}

The expression of Sirt1 decreased to $54.92 \pm 7.80 \%$ compared to donor hearts (Figure 1A). In advanced heart failure, atrial myocyte showed some hypertrophy and disarragement as expected (Hematoxylin-eosin staining in Figure 1B). Sirt1's expression as observed using immunohistochemistry showed reduced nuclear expression (decreased to $52.74 \pm 12.72 \%$ compared to donor) with advanced heart failure (Figure 1B). This result is similar to those observed in changes to the aging murine hearts (Figure 1C).

\section{MnSOD, Trx1, and apoptotic proteins are modulated in advanced heart failure}

The expression of downstream targets of Sirt1, such as Mn-superoxide dismutase (MnSOD) which is modulated by Sirt1 [14], Trx1, and the molecules associated with apoptotic signaling pathway were analyzed using immunoblot. The expressions of beneficial molecules, such as MnSOD, Trx1 and Bcl-xL (0.71 \pm 0.02 -fold, $0.61 \pm 0.05$-fold, and $0.53 \pm 0.08$-fold vs. donor, respectively) are all decreased in advanced heart failure (Figure $2 \mathrm{~A}$ and $\mathrm{B}$ ). The expression of Bax was significantly increased $(1.62 \pm 0.18$-fold vs. donor) in advanced heart failure (Figure $2 \mathrm{~A}$ and $\mathrm{B}$ ).

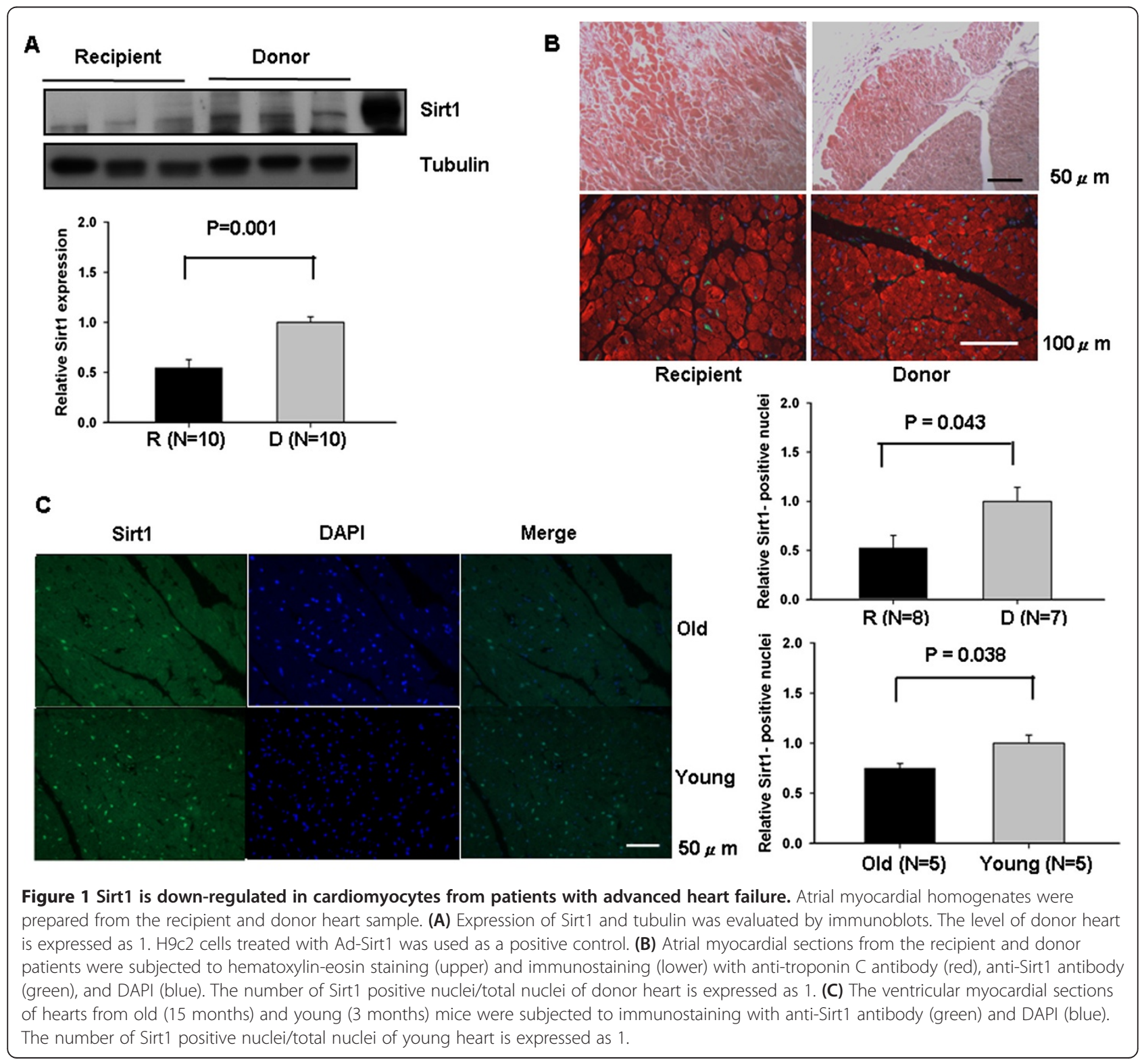


A
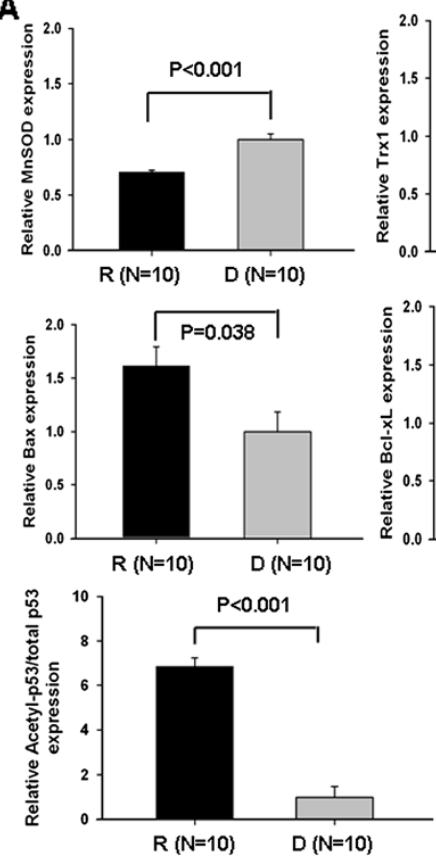

B
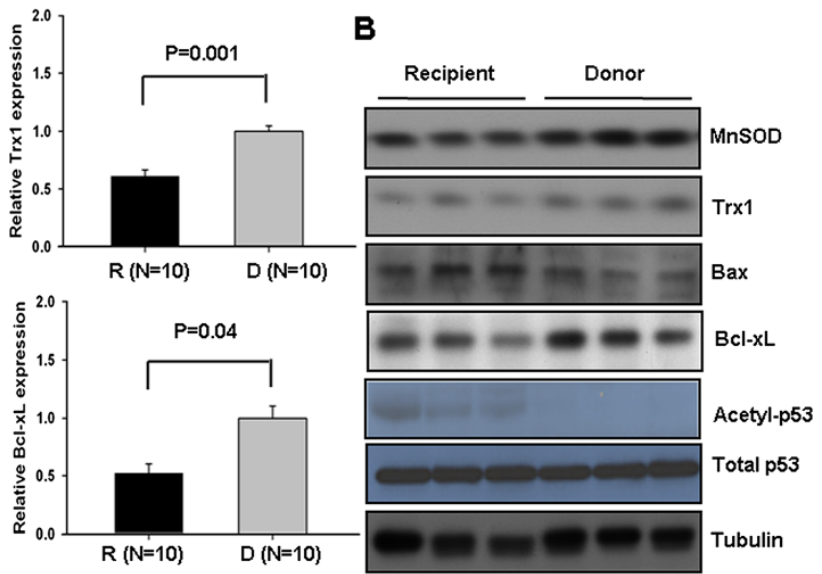

Figure 2 MnSOD, Trx1, and apoptotic proteins are modulated in advanced heart failure. Atrial myocardial homogenates were prepared from the recipient and donor heart sample. (A) Expression of MnSOD, Trx1, Bax, BCl-xL, acetyl-p53, p53 (total) and tubulin was evaluated by immunoblots. The level of donor heart is expressed as 1. (B) Representative immunoblots.

TUNEL-positive cardiomyocyte and oxidative stress are increased in advanced heart failure

TUNEL staining for both recipient and donor hearts was performed to further investigate changes associated with aging. The number of TUNEL positive cardiomyocytes in the heart failure group was increased significantly compared with normal donor control cardiomyocytes (Figure 3A). Due to the lower expression of Trx1 in advanced heart failure, differences in oxidative stress of the myocardium were further examined. 8-hydroxydeoxyguanosine (8-OHdG), a critical marker of oxidative damage of genomic DNA and the aging process $[15,16]$, was characterized using immunohistochemistry. Results showed increased oxidative stress in both nucleus and cytosol of cardiomyocytes in advanced heart failure, similar to the changes observed in aged cardiomyocytes (Figure 3B and C).

The activity of the AMPK-Nampt-Sirt1 axis is decreased in the advanced heart failure

Since both AMPK and Nampt are the upstream molecules of the functional signaling pathway of the AMPKNampt-Sirt1 axis [17], we examined their expression in advanced heart failure. Whether the axis of AMPKNampt-Sirt1 was functional in the heart was determined under the treatment of compound $\mathrm{C}$, an AMPK inhibitor. The expression of both Nampt and Sirt1 was decreased in $\mathrm{H} 9 \mathrm{c} 2$ cells, which implied that the axis may work in the heart (Figure 4A). The expression of both AMPK and Nampt was also decreased (0.60 \pm 0.06 -fold and $0.52 \pm 0.08$-fold vs. donor, respectively) in advanced heart failure as Sirt1's expression (Figure 4B). Furthermore, the expression of phospho-AMPK was severely inhibited in advanced heart failure (Figure 4B). In order to confirm these results, hearts from an aging C57/B6 mouse was examined. The expression of AMPK, Sirt1 and MnSOD was decreased in the old mice (aged 12 months) compared with younger mice (3 months), but the expression of Nampt was increased. The expression of phosphoAMPK/total AMPK was also checked and showed significantly decreased (or inhibited) in the old mice (aged 12 months) (Figure 4C).

\section{Translocation of Sirt1 and regulation of FoxO1 and p53 in} advanced heart failure

Due to lower expression of Sirt1 and MnSOD in heart failure, translocation of transcriptional factor FoxO1, which could modulate the expression of MnSOD [18], was examined, Immunostaining revealed decreased FoxO1 in the nucleus in advanced heart failure cardiomyocytes (Figure 5A). Furthermore, because of the increased expression of Bax, a downstream effector that is positively regulated by p53 in heart failure [19], we examined acetylated p53, which was significantly increased in advanced heart failure (Figure 5B). 


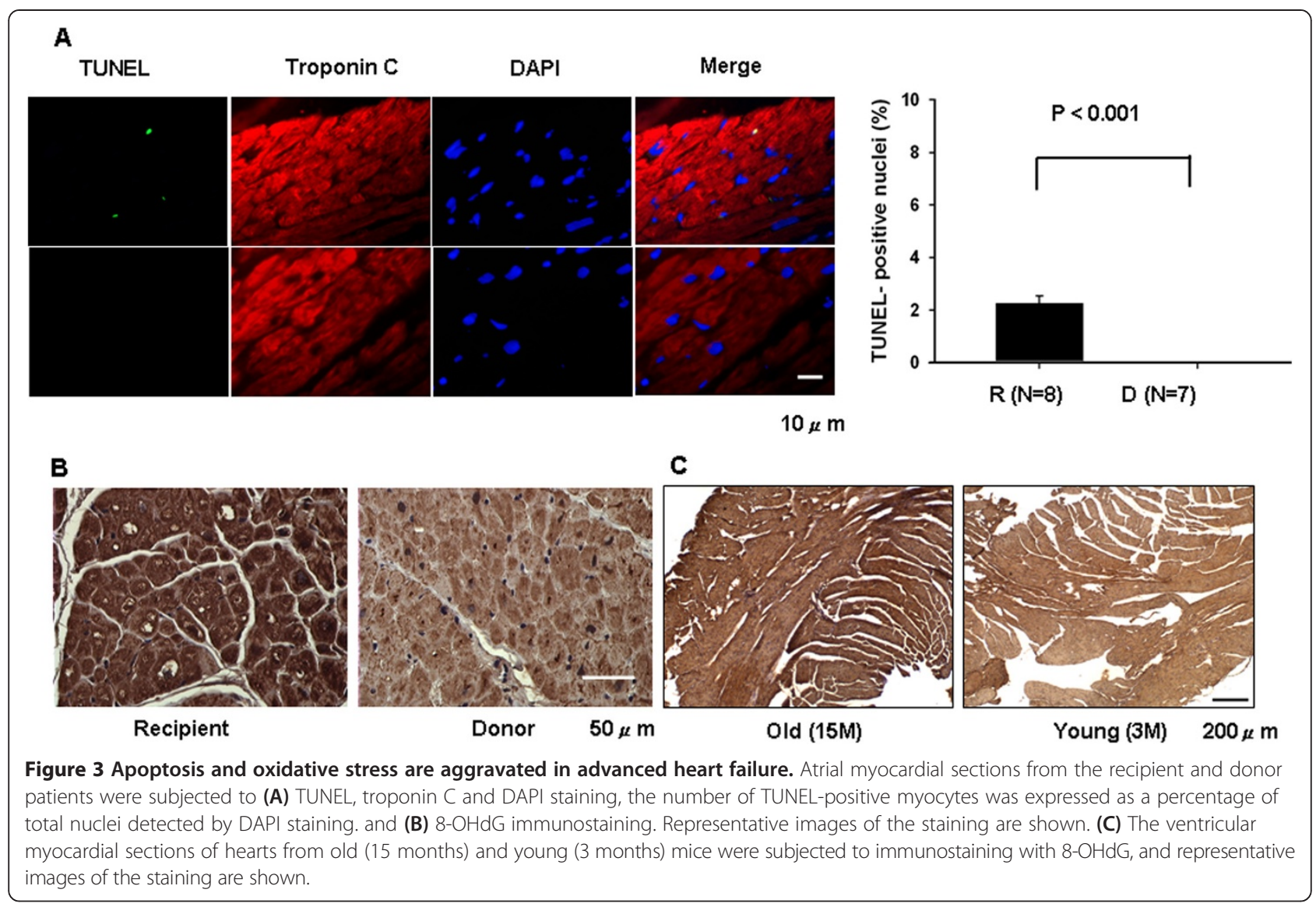

\section{Discussion}

This is a cross-sectional study designed to investigate the expression of Sirt1 and its associated molecules in the left atrium from the end-stage heart failure patients, and compared those from even younger donors. Simultaneously, these changes in heart failure are found to be similar to those observed in aging mice. Both of our study and Sakamoto et al's, [9] showed that the expression of Sirt1 in the heart is decreased with age. The gap in age, in spite of statistical insignificance, may have some minor effect on the Sirtl's expression. Hence, we checked whether the expression of Sirt1 is affected by aging effect in our sample (Additional file 1: Figure S1). It showed that it could be neglected, and the observations in this study are mostly related to progressive genetic remodeling of the failing heart.

It is widely agreed that Sirt1 plays a cardioprotective role in many aspects. There have been various studies conducted on in vivo heart models [11,20], ex vivo Langendorff model $[11,20,21]$, or cultured cardiomyocytes [10,12,22], which all suggested that Sirt1 serves a protective role against cardiovascular stresses through the regulation of apoptosis inhibition, aging retardation, and oxidative stress alleviation $[6,8,10]$.
We currently demonstrate that Sirt1 is down-regulated in the myocardium in patients with advanced heart failure, The result, as was supposed, is very similar to our previous report [11], and consistent with the report of human samples by Pillai et al. [13]. Alcendor et al. [10], showed that Sirt1 is up-regulated (8.8-fold of protein expression) after 4 weeks of pressure overload in mice, a condition used to represent the stage of heart failure, however, they did not check the cardiac function of these mice, nor the activity of Sirt1. Increase of Sirt1 expression in the heart was associated with cardiac hypertrophy $[10,23]$, however, the constitutive high level of Sirt1 may be harmful to the heart itself and reduce cardiac function, proved by transgenic model $[10,24]$. It also points out that the expression level of Sirt1 is important, too much is as bad as too little. In our study, we found that Sirt1 is obvious decreased in the heart of 12 or 15 month mice, and a significant decline in left ventricular systolic function was not noted until 18 months of age in the C57BL/6J mice [25], the same background of mouse as our study. Taken above findings altogether, it might be assumed that Sirt1 may be highly up-regulated to protect the cardiomyocytes in the early stage of heart failure (compensated stage) associated with pressure- 


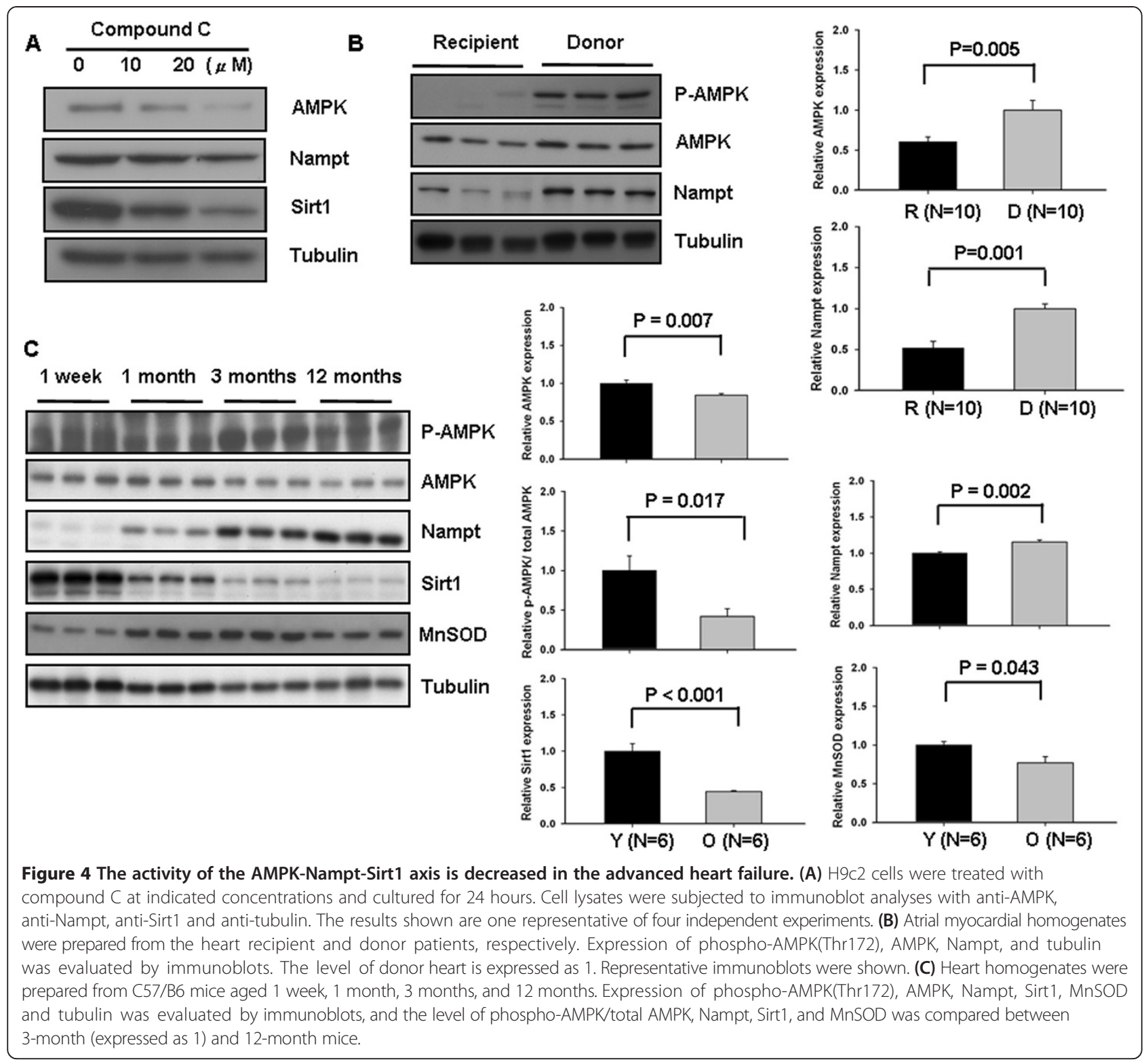

overload, which could be explained the finding of elevation of Sirt1's expression in dog by Alcendor et al. [12], however, the sustained too high level of Sirt1 is not beneficial, but detrimental to the cardiomyocytes through unclear mechanisms and the expression of Sirt1 may be progressively decreased accompanying the injury of cardiomyocytes in the long run (decompensated stage), and contributes partly to heart failure finally. More experiments are necessary to investigate the hypothesis. Furthermore, the reason for the discrepancy about higher expression of Sirt1 in old monkeys by Alcendor et al. [10] remains to be elucidated, and whether any stress impacted on these old monkeys or not should be explored.

We have previously reported that Sirt1 can stimulate the expression of MnSOD in cultured cardiomyocytes, for which FoxO1 has been shown to play an essential role [11], and in response to serum starvation in vitro, the deacetylation of p53 by Sirt1 inhibits cardiac myocytes apoptosis [12]. We currently report that in advanced heart failure, both the FoxO1 and deacetylation of p53 were significantly reduced in the nuclei of cardiomyocytes. These may be the mechanisms of reduced MnSOD, $\mathrm{Bcl}-\mathrm{xL}$, and increased Bax, apoptosis, and oxidative stress with the downregulation of Sirt1 expression.

Regardless of the stressor, downregulation of Sirt1 in the heart was associated with loss of cardioprotection in the literatures, such as our result that low expression of Sirt1 in advanced heart failure may lead to additional irreversible heart injury via downstream effectors. However, further studies are necessary to fully elucidate the 


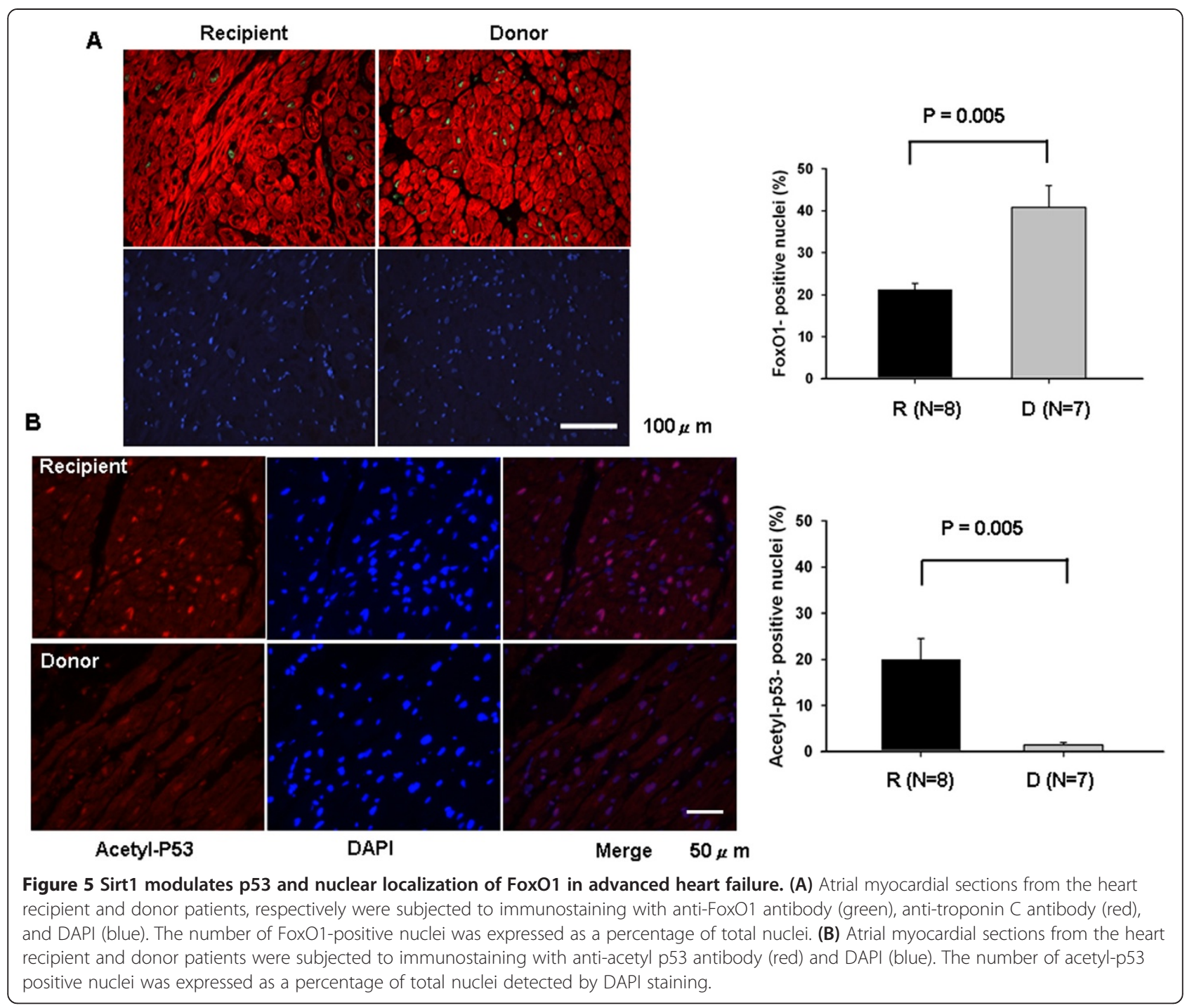

mechanism of how Sirt1 down-regulated, especially the relationship between Sirt1 and mechanical or neurohormonal stimulus of the failing heart before we target it.

Sirtuin activity is regulated by $\mathrm{NAD}^{+}$biosynthetic pathways, in which Nampt plays a critical role as a regulator for $\mathrm{NAD}^{+}$synthesis in cardiomyocytes [26]. Fulco et al. showed that the axis of AMPK-Nampt-Sirt1 was a functional pathway to sense and react to nutrient availability in skeletal muscle cells [17]. We also examine it in our samples and find the expression of Nampt is inconsistent in aged murine hearts and advanced heart failure. In aged heart, the expression of Nampt is increased, which may be secondary to compensate for the decrease of Sirt1. Moreover, in the mouse sample, the expression of phospho-AMPK and MnSOD is not synchronous to that of Sirt1 in the early age. The activation of phospho-AMPK in the early neonatal life may be likely associated with starvation (fasting) after birth, and it may imply that the regulation of these molecules is more complicated than our mention.

Our result suggests that the AMPK-Nampt-Sirt1 pathway may be disordered in heart failure and the mechanism of aging and heart failure is similar but not exactly the same. It also implicates that multiple molecular pathways involved in determining aging process. Recently, Ma et al. [27] also showed that ischemic AMPK activation was impaired in aged murine hearts, and Reznick et al. [28] disclosed that aging-associated reductions in AMPK activity may be an important contributing factor in reduced mitochondrial function and dysregulated intracellular lipid metabolism. We also report similar results of AMPK change in aged murine hearts. Per results provided by Fulco et al. glucose restriction (or nutrient shortage) functions to keep skeletal myoblasts from differentiation and hold them in the younger (undifferentiated) stage through activated AMPK. Although the exact 
molecular mechanisms through which nutrients influence various cell signaling/modulators of lifespan remain a largely unresolved issue. However, the above findings showed that the AMPK and/or activated AMPK, and the modulated Sirt1, may be regarded as important regulators or markers of aging, even the differentiation of cardiomyocytes. Upregulation of the functioning pathway can be an alternative method apart from increasing Sirt1's activity or expression, as a cardioprotective intervention. The metabolism of nutrition in the myocardium will change substantially during advanced heart failure, however, further experiments are necessary to elucidate the role of Sirt1 on the metabolic remodeling of the failing heart.

\section{Clinical relevance}

Downregulation of Sirt1 may have important meaning in the advanced heart failure, though cause and effect relationship is not yet established in our observation. Sirt1 agonists, such as Resveratrol, has been shown to protect the heart from ischemia/reperfusion [29]. Recently, Telmisartan, an angiotensin receptor blocker used in the management of hypertension or heart failure, was found to activate the AMPK-Sirt1 pathway in skeletal muscle, and ameliorate insulin sensitivity of obese $\mathrm{db} / \mathrm{db}$ mice [30]. These Sirt1 agonists may prevent changes associated with cardiac aging, and targeting Sirt1 may become a promising modality to protect the heart from aging or heart failure.

\section{Limitation}

There are several limitations in the study. One is because of impossibility to obtain the ventricular sample from the donor heart, we only used left atrium samples instead of left ventricle. Even though, we can not get the similar region from left atrium between donor and recipient heart while heart transplantation. Another is that the cause of heart failure is dilated cardiomyopathy in our study, younger than congestive heart failure due to ischemic heart disease, hypertension, or valvular heart disease. The observations may not be applied completely to other causes of congestive heart failure. Further investigations may be necessary to explore the difference.

\section{Conclusions}

In conclusion, in advanced heart failure, the changes associated with aging may be accelerated and the cardioprotective effect of Sirt1 is reduced due to the lower expression level and changes to downstream effector molecules. In addition, the reduced overall expression of AMPK-Nampt-Sirt1 may partly explain the molecular basis of this mechanism.

\section{Additional file}

Additional file 1: Figure S1. The regression analysis of relative expression of Sirt1 and age of patient in our sample. The coefficient of determination (R-Square) of both donor and recipient sample is 0 .

\section{Competing interests}

The authors declare that they have no competing interests.

\section{Authors' contributions}

T-ML and J-YT carried out the molecular studies. J-YT drafted the manuscript. Y-CC carried out the immunostainings. C-YH performed the statistical analysis, H-LH and C-FW perfromed the sample harvesting and preparation. T-ML participated in its design. C-CS and C-PH conceived of the study, and participated in its design and coordination and helped to draft the manuscript. All authors read and approved the final manuscript.

\section{Acknowledgements}

This work was partly assisted by the Division of Experimental Surgery of the Department of Surgery and the Animal Center of Department of Medical Research and Education Taipei Veterans General Hospital. This work was supported by the grant: NSC100-2314-B-075-014-MY2 from the National Science Council, Taiwan; V101C-018, and V102C-032 from the Taipei Veterans General Hospital, Taiwan.

\section{Author details}

${ }^{1}$ National Yang-Ming University, Institute of Clinical Medicine, School of Medicine, Taipei, Taiwan. ${ }^{2}$ Division of Cardiology, Department of Internal Medicine, Taipei Veterans General Hospital, Taipei, Taiwan. ${ }^{3}$ Division of Cardiovascular Surgery, Department of Surgery, Taipei Veterans General Hospital, 201, Section 2, Shih-Pai Road, Taipei 112, Taiwan. ${ }^{4}$ Department of Pathology, National Yang-Ming University Hospital, Yi-Lan, Taiwan.

${ }^{5}$ Department of Cardiovascular Surgery, Far Eastern Memorial Hospital, New Taipei, Taiwan.

Received: 7 December 2013 Accepted: 21 May 2014

Published: 9 June 2014

\section{References}

1. Rosamond W, Flegal K, Friday G, Furie K, Go A, Greenlund K, Haase N, Ho M, Howard V, Kissela B, Kittner S, Lloyd-Jones D, McDermott M, Meigs J, Moy C, Nichol G, O'Donnell CJ, Roger V, Rumsfeld J, Sorlie P, Steinberger J, Thom T, Wasserthiel-Smoller S, Hong Y: Heart disease and stroke statistics-2007 update: a report from the American heart association statistics committee and stroke statistics subcommittee. Circulation 2007, 115(5):e69-e171.

2. Nadal-Ginard B, Kajstura J, Anversa P, Leri A: A matter of life and death: cardiac myocyte apoptosis and regeneration. J Clin Invest 2003, 111 (10):1457-1459.

3. Lakatta EG, Sollott SJ: Perspectives on mammalian cardiovascular aging: humans to molecules. Comp Biochem Physiol A Mol Integr Physiol 2002, 132(4):699-721.

4. Guarente L: Sir2 links chromatin silencing, metabolism, and aging. Genes Dev 2000, 14(9):1021-1026.

5. Ha CW, Huh WK: The implication of Sir2 in replicative aging and senescence in saccharomyces cerevisiae. Aging (Albany NY) 2011, 3(3):319-324.

6. Howitz KT, Bitterman KJ, Cohen HY, Lamming DW, Lavu S, Wood JG, Zipkin RE, Chung P, Kisielewski A, Zhang LL, Scherer B, Sinclair DA: Small molecule activators of sirtuins extend Saccharomyces cerevisiae lifespan. Nature 2003, 425(6954):191-196.

7. Tissenbaum HA, Guarente L: Increased dosage of a sir-2 gene extends lifespan in caenorhabditis elegans. Nature 2001, 410(6825):227-230.

8. Wood JG, Rogina B, Lavu S, Howitz K, Helfand SL, Tatar M, Sinclair D: Sirtuin activators mimic caloric restriction and delay ageing in metazoans. Nature 2004, 430(7000):686-689.

9. Sakamoto J, Miura T, Shimamoto K, Horio Y: Predominant expression of Sir2alpha, an NAD-dependent histone deacetylase, in the embryonic mouse heart and brain. FEBS Lett 2004, 556(1-3):281-286. 
10. Alcendor RR, Gao S, Zhai P, Zablocki D, Holle E, Yu X, Tian B, Wagner T, Vatner SF, Sadoshima J: Sirt1 regulates aging and resistance to oxidative stress in the heart. Circ Res 2007, 100(10):1512-1521.

11. Hsu CP, Zhai P, Yamamoto T, Maejima Y, Matsushima S, Hariharan N, Shao D, Takagi H, Oka S, Sadoshima J: Silent information regulator 1 protects the heart from ischemia/reperfusion. Circulation 2010, 122(21):2170-2182.

12. Alcendor RR, Kirshenbaum LA, Imai S, Vatner SF, Sadoshima J: Silent information regulator 2alpha, a longevity factor and class III histone deacetylase, is an essential endogenous apoptosis inhibitor in cardiac myocytes. Circ Res 2004, 95(10):971-980.

13. Pillai JB, Isbatan A, Imai S, Gupta MP: Poly(ADP-ribose) polymerase-1dependent cardiac myocyte cell death during heart failure is mediated by NAD + depletion and reduced Sir2alpha deacetylase activity. J Biol Chem 2005, 280(52):43121-43130.

14. Pfluger PT, Herranz D, Velasco-Miguel S, Serrano M, Tschop MH: Sirt1 protects against high-fat diet-induced metabolic damage. Proc Natl Acad Sci U S A 2008, 105(28):9793-9798.

15. Cheng KC, Cahill DS, Kasai H, Nishimura S, Loeb LA: 8-Hydroxyguanine, an abundant form of oxidative DNA damage, causes G--T and A--C substitutions. J Biol Chem 1992, 267(1):166-172.

16. Mecocci P, Fano G, Fulle S, MacGarvey U, Shinobu L, Polidori MC, Cherubini A, Vecchiet J, Senin U, Beal MF: Age-dependent increases in oxidative damage to DNA, lipids, and proteins in human skeletal muscle. Free Radic Biol Med 1999, 26(3-4):303-308.

17. Fulco M, Cen Y, Zhao P, Hoffman EP, McBurney MW, Sauve AA, Sartorelli V: Glucose restriction inhibits skeletal myoblast differentiation by activating SIRT1 through AMPK-mediated regulation of Nampt. Dev Cell 2008, 14(5):661-673.

18. Kops GJ, Dansen TB, Polderman PE, Saarloos I, Wirtz KW, Coffer PJ, Huang $\Pi$, Bos $J$, Medema RH, Burgering BM: Forkhead transcription factor FOXO3a protects quiescent cells from oxidative stress. Nature 2002, 419(6904):316-321.

19. Miyashita T, Reed JC: Tumor suppressor p53 is a direct transcriptional activator of the human bax gene. Cell 1995, 80(2):293-299.

20. Tong C, Morrison A, Mattison S, Qian S, Bryniarski M, Rankin B, Wang J, Thomas DP, Li J: Impaired SIRT1 nucleocytoplasmic shuttling in the senescent heart during ischemic stress. FASEB J 2013, 27(11):4332-4342.

21. Nadtochiy SM, Redman E, Rahman I, Brookes PS: Lysine deacetylation in ischaemic preconditioning: the role of SIRT1. Cardiovasc Res 2011, 89(3):643-649.

22. Becatti M, Taddei N, Cecchi C, Nassi N, Nassi PA, Fiorillo C: SIRT1 modulates MAPK pathways in ischemic-reperfused cardiomyocytes. Cell Mol Life Sci 2012, 69(13):2245-2260.

23. Li L, Zhao L, Yi-Ming W, Yu YS, Xia CY, Duan JL, Su DF: Sirt1 hyperexpression in SHR heart related to left ventricular hypertrophy. Can J Physiol Pharmacol 2009, 87(1):56-62.

24. Kawashima T, Inuzuka Y, Okuda J, Kato T, Niizuma S, Tamaki Y, Iwanaga Y, Kawamoto A, Narazaki M, Matsuda T, Adachi S, Takemura G, Kita T, Kimura T, Shioi T: Constitutive SIRT1 overexpression impairs mitochondria and reduces cardiac function in mice. J Mol Cell Cardiol 2011, 51(6):1026-1036.

25. Boyle AJ, Shih H, Hwang J, Ye J, Lee B, Zhang Y, Kwon D, Jun K, Zheng D, Sievers $R$, Angeli $F$, Yeghiaarians $Y$, Lee $R$ : Cardiomyopathy of aging in the mammalian heart is characterized by myocardial hypertrophy, fibrosis and a predisposition towards cardiomyocyte apoptosis and autophagy. Exp Gerontol 2011, 46(7):549-559.

26. Hsu CP, Oka S, Shao D, Hariharan N, Sadoshima J: Nicotinamide phosphoribosyltransferase regulates cell survival through NAD + synthesis in cardiac myocytes. Circ Res 2009, 105(5):481-491.

27. Ma H, Wang J, Thomas DP, Tong C, Leng L, Wang W, Merk M, Zierow S, Bernhagen J, Ren J, Bucala R, Li J: Impaired macrophage migration inhibitory factor-AMP-activated protein kinase activation and ischemic recovery in the senescent heart. Circulation 2010, 122(3):282-292.

28. Reznick RM, Zong H, Li J, Morino K, Moore IK, Yu HJ, Liu ZX, Dong J, Mustard KJ, Hawley SA, Befroy D, Pypaert M, Hardie DG, Young LH, Shulman $\mathrm{Gl}$ : Aging-associated reductions in AMP-activated protein kinase activity and mitochondrial biogenesis. Cell Metab 2007, 5(2):151-156.
29. Sato M, Maulik N, Das DK: Cardioprotection with alcohol: role of both alcohol and polyphenolic antioxidants. Ann N Y Acad Sci 2002, 957:122-135.

30. Shiota A, Shimabukuro M, Fukuda D, Soeki T, Sato H, Uematsu E, Hirata Y, Kurobe H, Maeda N, Sakaue H, Masuzaki H, Shimomura I, Sata M: Telmisartan ameliorates insulin sensitivity by activating the AMPK/SIRT1 pathway in skeletal muscle of obese $\mathrm{db} / \mathrm{db}$ mice. Cardiovasc Diabetol 2012, 11:139.

doi:10.1186/1423-0127-21-57

Cite this article as: Lu et al: Downregulation of Sirt1 as aging change in advanced heart failure. Journal of Biomedical Science 2014 21:57.

\section{Submit your next manuscript to BioMed Central and take full advantage of:}

- Convenient online submission

- Thorough peer review

- No space constraints or color figure charges

- Immediate publication on acceptance

- Inclusion in PubMed, CAS, Scopus and Google Scholar

- Research which is freely available for redistribution 